\title{
Growth of Au Particles Evaporated on KBr and KCl Substrates Wearing Increased Densities of Surface Steps*
}

\author{
Yuuko FUKAZAWA*1, Suguru SHIBATA*1, Masatake IKEMOTO*1, \\ Hiroki KAWATOKO ${ }^{* 1}$ and Yasufumi SUSUKI*1 \\ ${ }^{*}$ Division of Science Education, Osaka-Kyoiku University, 4-698-1 Asahigaoka, Kashiwara-shi, Osaka 582-8582, Japan
}

(Received November 24, 2013, Accepted January 7, 2014)

Growth forms of Au particles prepared by vacuum evaporation methods on $\operatorname{KBr}(001)$ and $\mathrm{KCl}(001)$ substrates with two types of surface steps prepared by thermal sublimation and by electron stimulated desorption are studied by using a carbon backing method with a transmission electron microscope. Spiral steps of monoatomic height have been prepared on the substrate surfaces by heat sublimation. Epitaxial Au particles with pyramidal shapes and other multiply twined particles are grown by the vacuum evaporation on the substrates at temperatures of $50-450^{\circ} \mathrm{C}$. The size distributions of the particles on the substrates are reported and the typical size is measured to be smaller than $20 \times 20 \mathrm{~nm}^{2}$. The ratio of populations of the multiply twined particles along the $\mathrm{KBr}$ steps is increased compared with that on the flat area. However, this increase in the ratio is not observed for particles grown on $\mathrm{KCl}$. Additionally, step assemblies are prepared by electron stimulated desorption (ESD). The dependence of the size distributions of the Au particles grown on the substrates on electron fluence is measured for the ESD surfaces. With increasing electron fluence, the peak particle distribution size becomes smaller and particles smaller than $1 \mathrm{~nm}$ are observed.

\section{Introduction}

Since the discovery of the catalytic action of $\mathrm{Au}$ nanoparticles on $\mathrm{TiO}_{2}$, nanoparticles and nanowires have attracted much interest ${ }^{1)}$. Mechanisms of reaction by the catalyst, optical properties of the nanoparticles and nanowires and electron transport properties of the nanowires have been vigorously investigated ${ }^{2-5}$. Additionally, particles smaller than $1 \mathrm{~nm}$, so-called clusters, are also worthy of study both for the understanding of the fundamental properties of nanoparticles and for their applications ${ }^{5)}$. The physical properties of the nanoparticles including their growth forms are measured mostly under clean vacuum conditions. In many cases, however, the $\mathrm{Au}$ nanoparticles and nanowires are made up in wet processes that may produce surfaces that are not as clean as those achieved under the vacuum conditions ${ }^{1,5)}$. Thus studying the growth forms of $\mathrm{Au}$ nanoparticles and nanowires in various vacuum conditions, particularly, in easy to achieve high vacuum conditions, is worthy from the point of view of quality and ease of application.

The properties of small metal particles grown on alkali halide crystals have been measured for the last 80 years $^{6-11)}$. In most early studies, growth of the particles was examined in traditional high vacuum systems. However, ultra-high vacuum systems have been used in the field from as early as $1962^{7)}$. During this period, a lot of data was presented by many groups with respectable vacuum conditions. In some early studies, it was found that small $\mathrm{Au}$ particles grown on alkali halide crystals

\footnotetext{
* Presented as a contributed poster at the 54th annual symposium of the Vacuum Society of Japan, Nov. 26, 2013
}

typically have two crystal forms ${ }^{7-11)}$. The first, octahedron, is epitaxially grown single crystal particles (EPs), and the second, decahedron, is multiply twined particles (MTPs). In addition, composite particles of them are also grown. The MTPs are reported to be easy to grow along the steps on a $\mathrm{KBr}$ (001) surface, however, on other substrates the MTPs are hard to grow along the steps $^{9,11)}$.

In the past several years, we have investigated surfaces of alkali halide crystals irradiated by electron fluences of $10^{14}-10^{16} \mathrm{~cm}^{-2}$. Our experimental measurements were carried out with an ion surface-channeling technique with aid of observation by atomic force microscopes (AFM). The surfaces have many overlapped steps of monoatomic height created by electron-stimulateddesorption (ESD) ${ }^{12}$. We expect that the steps can fix the Au clusters on the surface as the Au particles grow. Thus the steps may be applied as size and shape controls of the Au particles.

In this work, growth forms of Au particles on $\mathrm{KBr}$ and $\mathrm{KCl}$ substrates with monolayer surface steps are studied with an aim to inform work on the formation of nanoparticles with a controlled size and shape in relatively convenient ways. The particles are prepared by vacuum evaporation under two vacuum conditions: high vacuum (HV) or ultra-high vacuum (UHV). On the substrate surface, spiral steps of monoatomic height are prepared by heat sublimation and densely overlapped steps are prepared by ESD. For the surfaces with spiral steps, size distributions of EPs and MTPs are measured with a variety of substrate temperatures. For the ESD surfaces, the dependence of the size distributions on the electron fluence is measured. The influence of the vacuum conditions on the growth of Au particles is also illuminated by 
comparing the measured results between the different vacuum conditions.

\section{Experimental Procedure}

The samples of $\mathrm{KBr}$ and $\mathrm{KCl}$ were cleaved along the (001) face in air. The cleaved sample was mounted on a sample holder. The sets of holders and quartz oscillators used to measure the film thickness were placed one-byone in $2 \times 10^{-4} \mathrm{~Pa} \mathrm{HV}$ evaporator (JEE-4X; JEOL), $1 \times$ $10^{-4} \mathrm{~Pa} \mathrm{HV}$ chamber equipped with an electron-gun and evaporator-electrodes, and $3 \times 10^{-8} \mathrm{~Pa}$ UHV chamber equipped with a sample-cleavage system, evaporatorelectrodes and an electron-gun. The temperature on the sample holders could be adjusted from room temperature up to $450^{\circ} \mathrm{C}$ and was monitored by thermo-couples. While the vacuum pump used to evacuate the UHV chamber was an ion-pump (PST200CX II; ULVAC), those used to evacuate the HV apparatuses were oil diffusion pumps with an alkyl-di-phenyl-ether oil (Neovac SY; Matsumura Oil). The cleaved surfaces used for the proceeding measurements were prepared in air for the $\mathrm{HV}$ apparatuses, however, the samples were cleaved in the UHV chamber to obtain clean surfaces for the measurements under the UHV condition.

The spiral steps were prepared on the surfaces by heat sublimation. By raising the surface temperature to $340^{\circ} \mathrm{C}$ for $\mathrm{KBr}$ and $400^{\circ} \mathrm{C}$ for $\mathrm{KCl}$ for 30 minutes, the steps could be easily formed. Au was deposited onto the surface in the HV evaporator or the UHV chamber at various temperatures $\left(50-400^{\circ} \mathrm{C}\right)$. The deposition rate of $\mathrm{Au}$ was in the range of $0.5-1 \mathrm{~nm} / \mathrm{min}$ and the mean film thicknesses evaporated were $1.5 \mathrm{~nm}$ for $\mathrm{KBr}$ substrates and $10 \mathrm{~nm}$ for $\mathrm{KCl}$ substrates. The angle between the evaporator-filament and the surface normal was less than $15^{\circ}$.

The ESD surfaces of $\mathrm{KBr}(001)$ for Au deposition were prepared in the $1 \times 10^{-4} \mathrm{~Pa} \mathrm{HV}$ chamber. The acceleration energy for electrons was in a range of $2.0-5.0 \mathrm{keV}$, and the accelerated electrons were line-scanned on the surface with an electro-magnet in order to achieve uniform irradiation. The angle of the electron beam measured from the surface normal was $30^{\circ}$. The electron currents measured by a Faraday cup with a secondary electron suppressor were typically 50 and $500 \mathrm{nA} / \mathrm{cm}^{2}$ on the sample surface for 2.5 and $5 \mathrm{keV}$ electrons, respectively. The electron fluence was varied in the range $1 \times$ $10^{14}-3 \times 10^{16} \mathrm{~cm}^{-2}$. The surface temperature during the electron irradiation was fixed to be $150^{\circ} \mathrm{C}$, while that during the deposition was varied in the range of $50-350^{\circ} \mathrm{C}$. The angle between the evaporator-window and the surface normal was $60^{\circ}$. The mean film thickness evaporated was $0.3 \mathrm{~nm}$ and the deposition rate was $0.2 \mathrm{~nm} / \mathrm{min}$, which were smaller than those on the surfaces with spiral steps.

The deposited substrates were cooled and moved to another $\mathrm{HV}$ evaporator (JEE-5B; JEOL) in order to be backed with carbon foil of thickness of about $40 \mathrm{~nm}$. After evaporating the carbon foil, the foils were set afloat on distilled water, mounted on grids of an electron microscope (TEM, JEM-2100; JEOL) and observed.
Apart from these depositions and observations of the $\mathrm{Au}$ particles, the substrate surfaces prepared with steps were observed in air by an AFM of Kobe university (Nano Navi II, E-sweep; SII nano technology).

\section{Results and discussion}

\subsection{Growth on surfaces with spiral steps}

Figure 1 shows an example of AFM images of the spiral steps decorated on the surfaces of $\mathrm{KBr}$ substrates heated in the $\mathrm{HV}$ evaporator. The incubation time for the high temperature, $370^{\circ} \mathrm{C}$, was 30 minutes. The height of individual steps measured in section profile of the spiral step are $1 \mathrm{ML}$. Although the sublimation of the substrates can be seen at $300^{\circ} \mathrm{C}$ both for $\mathrm{KBr}$ and $\mathrm{KCl}$ resulting in circular holes of monolayer height, the spiral steps can be observed at temperatures above $340^{\circ} \mathrm{C}$ for $\mathrm{KBr}$ and $400^{\circ} \mathrm{C}$ for $\mathrm{KCl}$, respectively. The results are consistent with those measured by ourselves in the UHV chamber and also by other groups measured on the surfaces cleaved in UHV conditions ${ }^{11)}$.

Figure 2(a) shows examples of TEM images of the Au particles grown on the spiral steps. Although the substrate $\mathrm{KCl}$ temperature during the deposition of $\mathrm{Au}$ was fixed to be $350^{\circ} \mathrm{C}$, the incubation temperatures for creating spiral steps were in the range of $400-450^{\circ} \mathrm{C}$. Au particles grew along the spiral steps and also on the flat area ${ }^{9}$. The particle density along the spiral steps was larger than that on the flat area. Both of the up and down sides of the spiral step were decorated by the particles. The aligned orientation of the particles on the steps was as shown in Fig. 2(b). A dependence on the incubation temperature was not seen in the range, where the spiral steps are observed. These results are consistent with particles grown in UHV conditions ${ }^{7,11)}$.

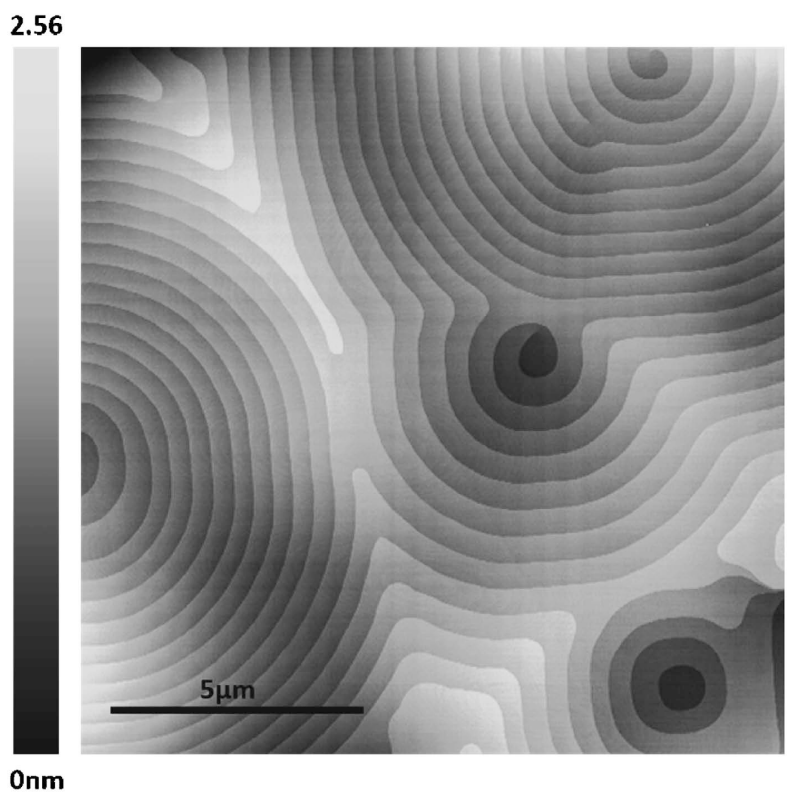

Fig. 1 An example of AFM images of the $\mathrm{KBr}$ surfaces heated under $\mathrm{HV}$ conditions. The incubation temperatures $(30$ minutes) was $370^{\circ} \mathrm{C}$. Spiral steps similar to these could be seen on $\mathrm{KCl}$ surfaces, however, rectangular lines along the spirals were comparatively more numerous. 

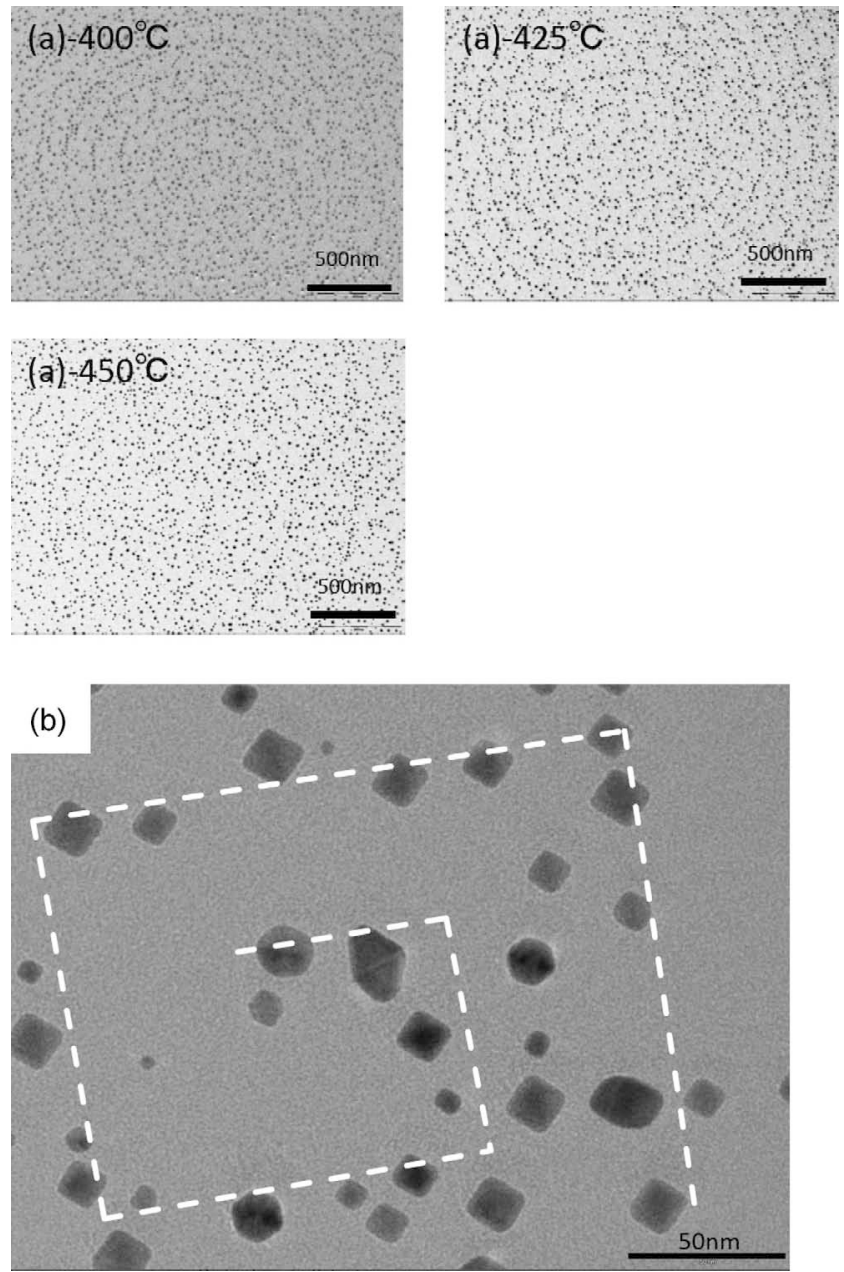

Fig. 2 (a) TEM images of the $\mathrm{Au}$ particles grown on $\mathrm{KCl}$ surfaces. The incubation temperatures (30 minutes) were 400, 425 and $450^{\circ} \mathrm{C}$, respectively. (b) An example of orientation of the $\mathrm{Au}$ particles grown along the spiral steps. The directionally aligned particles are indicated by a broken rectangular spiral for an incubation temperature of $400^{\circ} \mathrm{C}$.

The distributions of the circle diameters containing polygonal Au particles (size distributions) grown on $\mathrm{KBr}$ and $\mathrm{KCl}$ surfaces decorated with spiral steps at an incubation temperature of $370^{\circ} \mathrm{C}(\mathrm{KBr})$ and $400^{\circ} \mathrm{C}(\mathrm{KCl})$ were measured for the various deposition temperatures. Although the most probable sizes of particles grown on $\mathrm{KBr}(001)$ surfaces increased with increasing deposition temperature in the range of $50-150^{\circ} \mathrm{C}$, they were almost independent of the deposition temperature in the range of $150-250^{\circ} \mathrm{C}$ and distributed between 15 and $17 \mathrm{~nm}$ in diameter. Those of particles grown on the $\mathrm{KCl}(001)$ were distributed between 10 and $20 \mathrm{~nm}$ in the temperature range of $150-400^{\circ} \mathrm{C}$.

It is well known that the EP particles show a square or rectangular shape in TEM images ${ }^{7,9,11)}$. In Fig. 3(a) and (b), the size distributions of EPs grown on $\mathrm{KBr}\left(250^{\circ} \mathrm{C}\right)$ and $\mathrm{KCl}\left(350^{\circ} \mathrm{C}\right)$ surfaces are shown. Almost all EPs are smaller than $20 \mathrm{~nm}$. It is also known that most of the MTPs show pentagonal, hexagonal or polygonal shapes in TEM images $9,11,13,14)$. The size distributions of the MTPs grown on $\mathrm{KBr}$ and $\mathrm{KCl}$ surfaces are shown in Fig. 3(c) and (d). The particles of incorporated polygons were
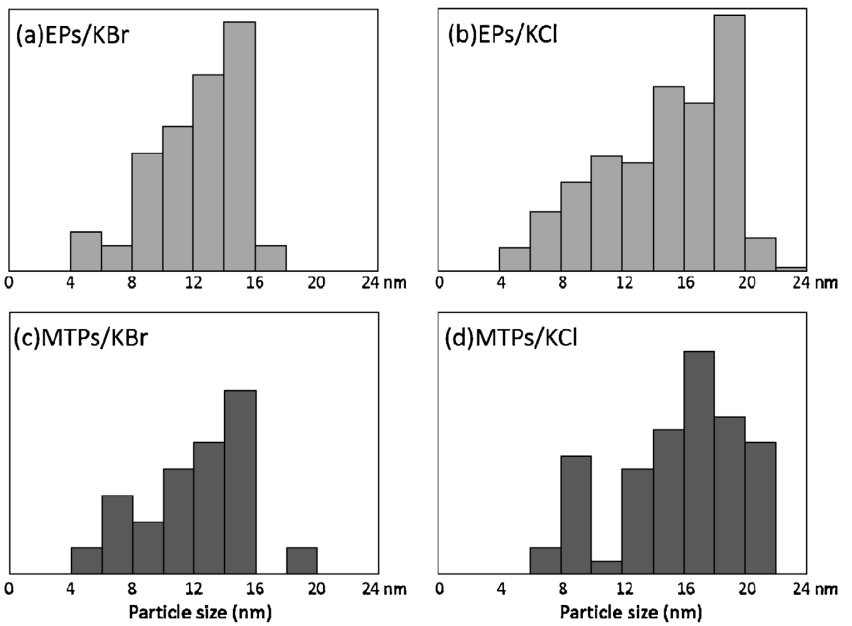

Fig. 3 Distributions of the circle diameters involving particles grown on the substrate surfaces. (a) Epitaxially grown single crystal particles (EPs) on $\mathrm{KBr}$ (b) EPs on $\mathrm{KCl}$ (c) Multiply twined particles (MTPs) on $\mathrm{KBr}$ (d) MTPs on $\mathrm{KCl}$. The sublimation incubation temperatures (30 minutes) were 370 and 400 ${ }^{\circ} \mathrm{C}$ for $\mathrm{KBr}$ and $\mathrm{KCl}$, respectively. The deposition temperatures were 250 and $350^{\circ} \mathrm{C}$ for $\mathrm{KBr}$ and $\mathrm{KCl}$, respectively.

regarded as composite particles. Comparing the distributions shown in Fig. 3, it can be seen that the size distributions of the EPs and MTPs are almost equal. These size distributions are not in disaccord with those for the particles grown under UHV conditions ${ }^{7,11)}$.

The ratios of EPs and MTPs grown along the spiral steps and on the flat area are shown in Fig. $\mathbf{4}$ for $\mathrm{KBr}$ and $\mathrm{KCl}$ substrates. In the measured temperature range, the ratio of populations of the MTPs along the steps of $\mathrm{KBr}$ is increased compared with that on the flat area as shown in Fig. 4(a) and (b). For KBr substrates, the ratios are in good agreement with the solid lines, which are the published data measured in a UHV condition by another group ${ }^{11)}$. However, this increase in the ratio was not seen for particles grown on $\mathrm{KCl}$ as shown in Fig. 4 (c) and (d). Similar results, conflicting with those on $\mathrm{KBr}$, were reported on $\mathrm{NaCl}$ surface in a UHV condition by other groups $s^{9,11)}$.

Inspecting the temperature dependence shown in Fig. 4(a) and (b), it is known that the ratio of EPs crosses over that of MTPs as the temperature increases. Although this result reproduces those measured in UHV, the present results indicate that the temperatures of the cross over points shift high from those measured in UHV. The temperature difference between the two vacuum conditions is large on the flat area.

Comparing the spiral steps worn on the $\mathrm{KBr}$ and $\mathrm{KCl}$ surfaces between the $\mathrm{HV}$ and $\mathrm{UHV}$ conditions, it is concluded that no explicit difference could be found. In addition, it is also found that the Au particles grown on spiral steps were not affected explicitly by the difference in the vacuum conditions.

\subsection{Growth on surfaces with densely packed steps by ESD}

Figure 5 shows the AFM images of the $\mathrm{KBr}$ surface irradiated by $2 \mathrm{keV}$ electrons in the $\mathrm{HV}$ chamber. The sur- 

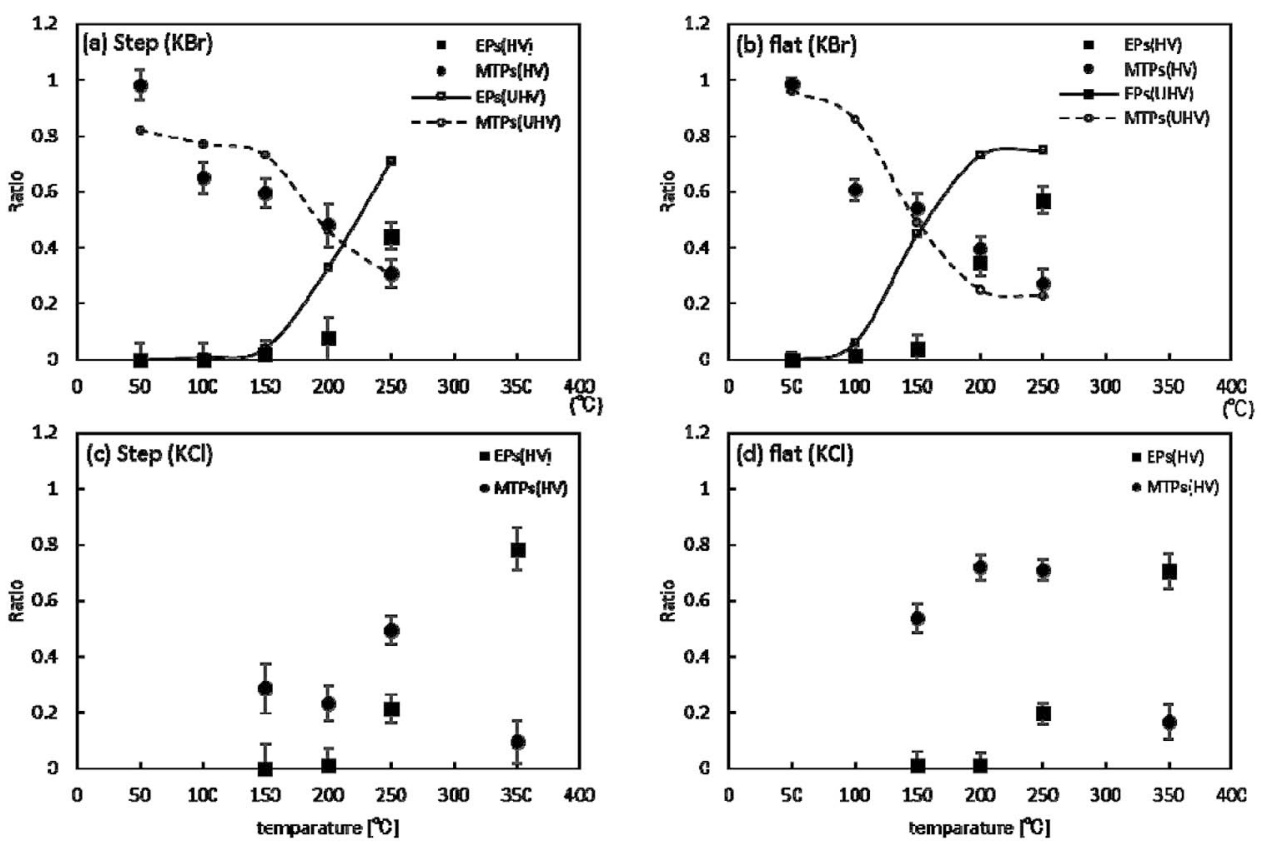

Fig. 4 Ratios of the epitaxially grown single crystal particles (EPs) and multiply twined particles (MTPs) grown along the spiral steps and on the flat area to the total number of particles for $\mathrm{KBr}$ and $\mathrm{KCl}$ surfaces. (a) and (b) Step and flat areas on $\mathrm{KBr}$ surfaces, respectively. For comparison, the solid and dashed curves are shown which are measured under UHV conditions were taken from ref. 11. (c) and (d) Step and flat areas on $\mathrm{KCl}$ surfaces, respectively.

6.0

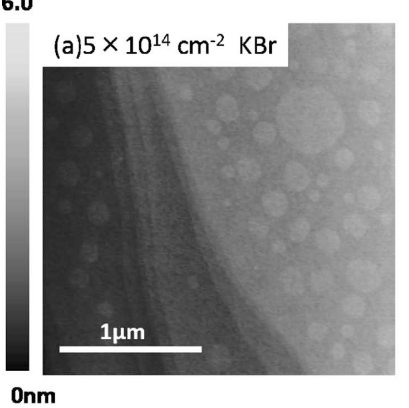

6.7

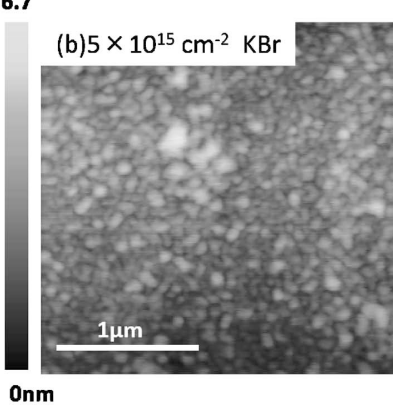

Fig. 5 AFM images of the $\mathrm{KBr}$ surfaces irradiated by $2 \mathrm{keV}$ electrons in the $\mathrm{HV}$ chamber. (a) $5 \times 10^{-14} \mathrm{~cm}^{-2}$. The striped steps seen on the left side are the cleavage steps. (b) $5 \times 10^{-15}$ $\mathrm{cm}^{-2}$.

face irradiated by $5 \times 10^{14} \mathrm{~cm}^{-2}$ electrons has circular pits of monolayer height and that irradiated by $5 \times 10^{15}$ $\mathrm{cm}^{-2}$ electrons has irregular overlapped steps. Although the observed morphologies of the surfaces irradiated in the HV chamber were not as clean as those irradiated in the UHV chamber, our previous studies under the UHV conditions show a minimal number of circular monolayer pits for overlapped steps in the fluence range of $10^{14}$ $\mathrm{cm}^{-2}$, and irregular pyramidal steps in the range of $10^{15}$ $\mathrm{cm}^{-2}{ }^{15}$. Thus the pyramidal steps result from overlap of rectangular monolayer pits by ESD ${ }^{15}$. The step density and especially the density of the overlapped steps increases with increasing electron fluence in the $10^{15} \mathrm{~cm}^{-2}$ range ${ }^{15,16)}$. In Fig. 5(b), the distinct overlapped steps are not seen, however, the increase of the step density can be seen.

Figure 6 shows the TEM images of $\mathrm{Au}$ particles deposited on $\mathrm{KBr}$ surfaces irradiated by 2.5 or $5 \mathrm{keV}$
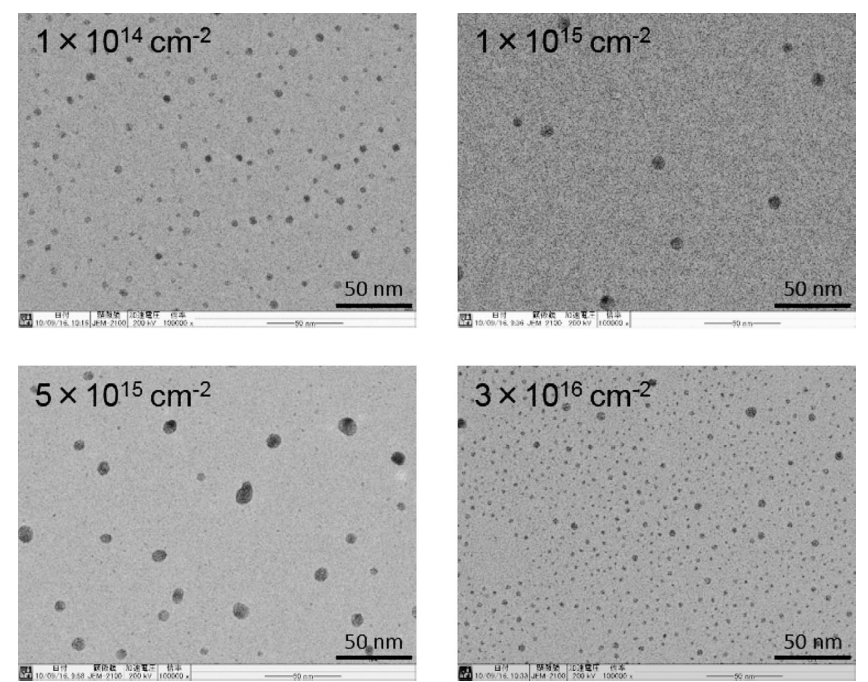

Fig. 6 TEM images of the $\mathrm{Au}$ particles deposited on the $\mathrm{KBr}$ surfaces irradiated by electrons.

electrons. The acceleration energies for electrons were 5 $\mathrm{keV}$ for an irradiation of $3 \times 10^{16} \mathrm{~cm}^{-2}$ and $2.5 \mathrm{keV}$ for others. The desorbed surface-atom yield for a $5 \mathrm{keV}$ electrons irradiation is expected to be twice that of a $2.5 \mathrm{keV}$ irradiation ${ }^{17)}$. Thus the morphology of the surface may depend on the electron energy and it is possible that the surfaces irradiated by $5 \mathrm{keV}$ electrons are strongly damaged compared with those irradiated by $2.5 \mathrm{keV}$ electrons.

It was found that there is a deposition temperature region, where the most probable sizes in the size distribution of $\mathrm{Au}$ particles are almost constant. This deposition temperature was $200-300^{\circ} \mathrm{C}$, which overlaps with the 


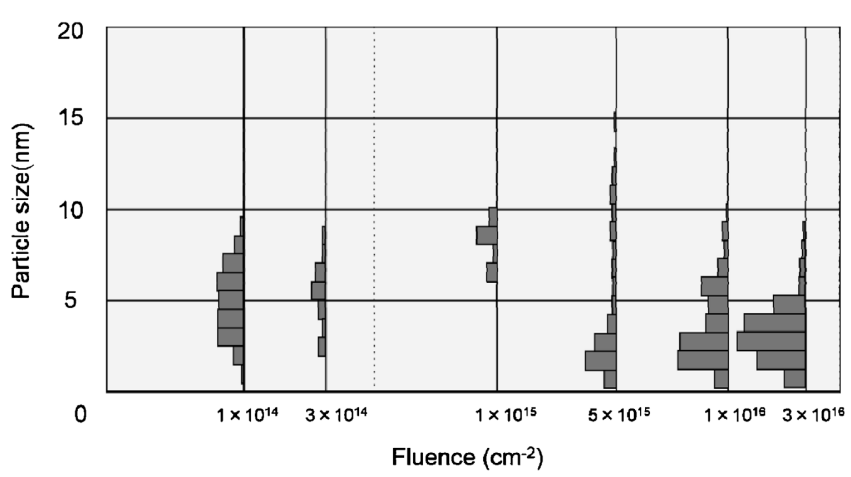

Fig. 7 Distributions of the diameter of particles grown on $\mathrm{KBr}$ surfaces for different electron fluences.

region measured for deposition on surfaces decorated with spiral steps. Therefore, the substrate temperature was fixed to be $250^{\circ} \mathrm{C}$. The sizes of the $\mathrm{Au}$ particles are expected to become smaller as the step density increases, i.e., as the irradiation fluence increases, since the steps work to impede the motion of the clusters grown on the surface. This result can be seen in Fig. 7, where the size distributions of the Au particles are shown for different electron fluences. The peak size of the distributions becomes slightly smaller as the fluence increases. For $5 \times$ $10^{15} \mathrm{~cm}^{-2}$, particles smaller than $1 \mathrm{~nm}$ are observed coexisting with those larger than $10 \mathrm{~nm}$. For fluences larger than $1 \times 10^{16} \mathrm{~cm}^{-2}$, the number of the particles larger than $10 \mathrm{~nm}$ decreases. Because of the small size of particles and the slightly larger thickness of the carbon foils evaporated to back these particles, we could not distinguish clearly the shapes of particles smaller than $5 \mathrm{~nm}$. Although both of EPs and MTPs can be seen in the images, most of these particles were not square in shapes for the surface irradiated with more than $5 \times 10^{15} \mathrm{~cm}^{-2}$. The high ratio of the MTPs supports that these particle's growth was influenced by the steps decorated on the surface by ESD.

Comparing the ESD surfaces shown in Fig. 5 with our previous results, the surfaces in the HV condition are relatively contaminated ${ }^{12,15)}$. We could not observe distinct overlapped pyramidal steps on these surfaces. However, the density of the overlapped steps increases with increasing electron fluence for the both vacuum conditions.

\section{Conclusion}

In this work, growth forms of $\mathrm{Au}$ particles near the steps on $\mathrm{KBr}$ and $\mathrm{KCl}$ substrates are studied by using vacuum evaporation methods. Particles along the spiral steps prepared by heat sublimation and on the assemblies of steps prepared by ESD were examined. Size and shape distributions of particles were measured with a variety of the substrate temperatures during the evaporation. For the spiral steps, the ratio of populations of the MTPs along the $\mathrm{KBr}$ steps is increased compared with that on the flat area. However, this increase of the ratio was not seen for particles grown on $\mathrm{KCl}$. As for the present systems treated, excepting the temperature shift of a few tens degrees, it was found that the different vacuum conditions did not show explicit effect. For the ESD surface, attention was given to the particle size distributions. The peaks of the size distributions become small with increasing electron fluence and the particles smaller than $1 \mathrm{~nm}$ start to be observed on surfaces with a fluence greater than $5 \times 10^{15} \mathrm{~cm}^{-2}$. The observed morphologies of the ESD surfaces irradiated in the HV chamber were not distinct from those irradiated under UHV conditions.

\section{Acknowledgements}

The authors wish to thank Professor Y. Fujii for the use of the AFM of Instrumental Analysis Division of Kobe University. The authors also wish to thank Professor K. Kandori for the use of the TEM of Center for Science Education of Osaka Kyoiku University. The authors are grateful to Asami Takase and Yohei Ueda for preparing the electron gun and preliminary experiments. The authors are also grateful to Yuki Okamoto, Hiroaki Umedzu, Tomohiro Maki and Takashi Fujii for their efforts in preparing the samples on the thermally sublimated surfaces of $\mathrm{KBr}$ and $\mathrm{KCl}$.

\section{References}

1) M. Haruta, N. Yamada, T. Kobayashi and S. Iijima: J. Catal., 115 (1989) 301.

2) M. S. Chen and D. W. Goodman: Science, 306 (2004) 252.

3) W. L. Barnes, A. Dereux and T, W. Ebbesen: Nature, 424 (2003) 824.

4) Y. Kondo and K. Takayanagi: Science, 289 (2000) 606.

5) Gold nanotechnology: Fundamentals and Applications, ed. M. Haruta (CMC publishing, Tokyo, 2009) [in Japanese].

6) H. Lassen: Phys. Z, 35 (1934) 179.

7) S. Ino, D. Watanabe and S. Ogawa: J. Phys. Soc. Jpn., 17 (1962) 1074.

8) H. Bethge: Surf. Sci., 3 (1964) 33.

9) H. Sato and S. Shinozaki: J. Appl. Phys., 41 (1970) 3165.

10) T. L. Gordillo and M. J. Yacaman: J. Vac. Sci. Tech., 15 (1978) 1195.

11) K. Yamamoto, T. Iijima, T. Kunishi, K. Fujiwara and T. Osaka: J. Cryst. Growth, 94 (1989) 629.

12) Y. Fukazawa, Y. Matsumoto, K. Mitsuhara and Y. Susuki: Appl. Surf. Sci., 256 (2009) 1184.

13) A. S. Barnard, X. M. Lin and L. A. Curtiss: J. Phys. Chem. B, 109 (2005) 24472.

14) L. D. Marks: J. Cryst. Growth, 61 (1983) 556.

15) Y. Matsumoto, Y. Fukazawa and Y. Susuki: J. Vac. Soc. Jpn., 52 (2009) 411 [in Japanese].

16) Y. Fukazawa, M. Ikemoto, S. Shibata and Y. Susuki: J. Vac. Soc. Jpn., 55 (2012) 167 [in Japanese].

17) Y. Fukazawa, K. Kihara, K. Iwamoto and Y. Susuki: J. Vac. Soc. Jpn., 56 (2013) 428. 